\title{
Current state of clinical development of TROP2-directed antibody-drug conjugates for triple-negative breast cancer
}

\author{
Maximilian Marhold (D)
}

Received: 10 October 2021 / Accepted: 18 November 2021 / Published online: 23 December 2021

(C) The Author(s) 2021

\begin{abstract}
Summary Antibody-drug conjugates (ADCs) against numerous molecular targets are currently being developed for the treatment of breast cancer (BCa). While the first ADC directed against Her2, namely trastuzumab-emtansine, was approved several years ago, targeting of TROP-2, an epithelial cell marker overexpressed in approximately $80 \%$ of triple-negative breast cancers (TNBC) has gained interest through positive clinical data reported for the compound sacituzumab-govitecan (SG) resulting from the phase 3 ASCENT trial. This short review summarizes the data that led to approval of SG and to take a closer look at the state of clinical development of other ADCs targeting TROP-2 in TNBC.
\end{abstract}

Keywords Breast neoplasm - Tumor-associated calcium-signal transducer 2 (TACSTD2) .

Sacituzumab-govitecan - Datopotamab-deruxtecan . ASCENT · ADC

\section{Introduction}

Triple-negative breast cancer (TNBC) is an aggressive variant of breast cancer that is defined by lack of expression of therapeutic targets. While various breakthroughs in treating TNBC have been reported in recent years-including the addition of immunotherapy using checkpoint inhibitors (CIs) such as atezolizumab [1] or pembrolizumab [2, 3] as well as targeted agents such as PARP inhibitors (PARPi) for BRCA-deficient TNBC patients $[4,5]$, early disease recurrence within 5 years after diagnosis remains

\footnotetext{
M. Marhold ( $\bowtie)$

Department of Medicine I, Clinical Division of Oncology,

Medical University Vienna, Währinger Gürtel 18-20,

1090 Vienna, Austria

maximilian.marhold@meduniwien.ac.at
}

common, with an approximate rate of $40 \%$ for distant and locoregional recurrence combined [6]. Recently, sacituzumab-govitecan (SG), an antibody-drug conjugate directed against TROP-2 (or tumor-associated calcium-signal transducer 2, TACSTD2, Chr. 1p32), which was first discovered in trophoblast cells and subsequently shown to affect cancer cell migration through anchorage-independent growth, showed clinical activity in the randomized phase 3 ASCENT trial in metastatic or locally advanced TNBC [7]. Based on the results of this confirmatory trial for prior data stemming from 108 patients within the IMMU-13201 phase $1 / 2$ basket study [8], the US Food and Drug Administration (FDA) granted SG approval. Although approval for Europe is still pending, it is likely that this novel therapeutic compound will become available shortly and probably define a new standard treatment for TNBC. With further studies of numerous compounds ongoing in various clinical settings, it is worthwhile discussing the current state of TROP-2directed therapy in TNBC.

TROP-2, also known as EpCAM2, was first described as a transmembrane trophoblast cell surface glycoprotein by Lipinski et al. in 1981 [9]. TROP-2 was shown to be overexpressed in certain cancers including TNBC, where its expression correlated with nodal status, metastasis and histological grade [10]. Its binding to a monoclonal antibody generated through injection of cell membrane lysate of a surgical squamous lung cancer specimen into immunocompetent $\mathrm{BALB} / \mathrm{c}$ mice in a preclinical study was-in retrospect-of high importance for the development of TROP-2-directed therapy [11, 12]. A humanized version of this IgG1 antibody, named RS7, was used as the base of SG [13].

Besides SG, further antibody-drug conjugates against TROP-2 were developed by various pharmaceutical companies. To my knowledge, only 
SG, datopotamab-deruxtecan (Dato-DXd, DS-1062), BAT8003 and SKB264 are still undergoing clinical evaluation.

\section{Sacituzumab-govitecan}

The approval of SG by the FDA was based on data from phase 1/2 IMMU-132-01 and phase 3 ASCENT trials. In both trials, objective response was seen in approximately one third of patients $33.3 \%$ in IMMU132-01, 35\% in ASCENT). Progression-free survival (PFS) in ASCENT was also found to be similar to that reported from IMMU-132-01, with patients receiving SG achieving 5.6 months. Interestingly, this translated into a meaningful clinical benefit in terms of overall survival (OS) when compared to the single agent chemotherapy control group, which consisted of physicians' choice of eribulin, vinorelbine, capecitabine or gemcitabine (12.1 vs. 6.7 months, hazard ratio $[\mathrm{HR}] 0.48)$. As most patients $(54 \%)$ had received eribulin in the control arm of ASCENT, it would be interesting to see SG tested against single compounds in earlier settings, e.g., capecitabine in second line. Still, as the benefit provided by SG was substantial and patients had received at least two prior lines of therapy, SG will undoubtedly change the standard of care in later line treatment of advanced TNBC. Side effects caused by govitecan or SN-38, the active metabolite of irinotecan linked to the antibody via a $\mathrm{pH}$-sensitive linker molecule, included neutropenia, diarrhea and nausea and should be part of a manageable toxicity profile in experienced hands. As the drug to antibody ratio (DAR) of SG was said to be relatively high at approximately 7.5 , side effects did not exceed expected rates [7]. Further development of SG in metastatic TNBC focuses on combination therapies with various immunotherapeutic substances such as the CIs pembrolizumab (phase 2 SACI-IO TNBC trial, NCT04468061) [14], atezolizumab (phase 1b/2 MORPHEUS-TNBC trial) [15] and avelumab (phase 2 InCITe trial, NCT03971409), or even cellular therapies such as PD-L1 targeting natural killer cells (t-haNK, NCT04927884) [16], as well as chemotherapeutics such as sabizabulin (phase 2, NCT05008510) and targeted agents such as the PARPi rucaparib (phase 1b/2 SEASTAR trial, NCT03992131) [17]. Lastly, a phase 2 trial evaluating activity of SG in patients suffering from HER2-negative brain metastasis is ongoing (SWOG S2007, NCT04647916).

Interestingly, beside its success in the metastatic setting, SG is also being evaluated in the (post)neoadjuvant and hence curative, early setting of TNBC treatment: The SASCIA trial (GBG 102, NCT04595565) is a phase 3 trial testing the use of SG in patients not achieving pathological complete response (pCR) after neoadjuvant treatment against physicians' choice of postneoadjuvant capecitabine, platinum-based therapy or observation in Her2-negative early BCa. Its main outcome measure will be invasive dis- ease-free survival (iDFS) [18]. The NeoSTAR trial (NCT04230109), on the other hand, is testing neoadjuvant activity of SG as monotherapy and in combination with the checkpoint inhibitor pembrolizumab. Standard chemotherapy regimen may be used followingly at the discretion of treating physicians. The primary outcome measure will be pathologic response [19].

\section{Datopotamab-deruxtecan}

DS-1062 or datopotamab-deruxtecan (Dato-DXd) is an ADC comprising a monoclonal anti-TROP-2 IgG1 molecule and the topoisomerase I inhibitor deruxtecan and is being developed by Daiichi Sankyo and Astra Zeneca. Its DAR was reported to be 4 , which is numerically lower compared to the ones reported for SG and trastuzumab-DXd (T-DXd; DAR of approximately 7-8) [20]. It is currently being investigated in the phase 1 TROPION-PanTumor01 trial, a basket trial including metastatic hormone receptor (HR)-positive and triple-negative breast cancer patients. After preclinical data had already shown activity in patient-derived xenograft models of non-small cell lung cancer (NSCLC) [21] and clinical data within the same entity have led to establishment of a phase 3 trial [22], first data from TROPION-PanTumor01 also indicated clinical activity in metastatic TNBC with an objective response rate (ORR) of $43 \%$ and disease control rate of $95 \%$ [23]. These data have to be considered immature, as recruiting was still ongoing when this short review was written. Nevertheless, this early report about clinical activity of Dato-DXd underlines the potential of targeting TROP-2 in TNBC. Furthermore, given the fact that deruxtecan has shown manageable toxicity as well as remarkable responses when combined with trastuzumab in advanced and pretreated HER2-positive breast cancer [20], the oncologic community has already gathered some experience with the cytotoxic part of this novel treatment, which could help in rapid rollout and safe use upon potential approval. Interestingly, the safety profile of Dato-DXd seems comparable to $\mathrm{T}-\mathrm{DXd}$, having said that contrary to the use of T-DXd, no cases of interstitial lung disease (ILD) were described for this compound in TROPION-PanTumor01 [23].

\section{Other ADCs targeting TROP-2 in TNBC}

SKB264, a TROP-2 directed ADC with the active cytotoxic agent of the topoisomerase I inhibitor belotecan and a reported DAR of 7.6 is being investigated in various unresectable or metastatic epithelial tumors including TNBC in a global open label multicenter phase $1 / 2$ trial (NCT04152499) [24]. No data on clinical activity in TNBC is available to date. The same is true for BAT8003, a maytansine derivativecarrying humanized antibody with reported DAR of 3.5. Analogous to SKB264, a phase I trial with cur- 
rently unknown recruitment status was initiated for BAT8003 (NCT03884517). Studies of other TROP-2 directed ADCs such as PF-06664178 (NCT02122146, [25]) were terminated due to toxicity or other concerns.

\section{Conclusion}

The concept of targeting of TROP-2 (TACSTD2, EpCAM2) using ADCs has been proven to ameliorate clinical outcomes of patients suffering from TNBC. Various ADCs from different pharmaceutical companies are currently under investigation in phase $1 / 2$ clinical trials while the ADC sacituzumab-govitecan has already been approved by the FDA based on data from the phase III ASCENT trial. Longer follow-up and larger trials will be needed to assess the impact of targeting TROP-2 in TNBC using ADCs.

Funding Open access funding provided by Medical University of Vienna.

Conflict of interest M. Marhold has received honoraria for lectures, advisory board participation and consultation from Roche, Eli Lilly, Novartis, AstraZeneca, Daiichi Sankyo, Pfizer and Medmedia and travel support from Amgen, Roche, Novartis, Pierre Fabre, Daiichi Sankyo, Pfizer and Eisai.

Open Access This article is licensed under a Creative Commons Attribution 4.0 International License, which permits use, sharing, adaptation, distribution and reproduction in any medium or format, as long as you give appropriate credit to the original author(s) and the source, provide a link to the Creative Commons licence, and indicate if changes were made. The images or other third party material in this article are included in the article's Creative Commons licence, unless indicated otherwise in a credit line to the material. If material is not included in the article's Creative Commons licence and your intended use is not permitted by statutory regulation or exceeds the permitted use, you will need to obtain permission directly from the copyright holder. To view a copy of this licence, visit http://creativecommons.org/licenses/by/4.0/.

\section{References}

1. Schmid P, Adams S, Rugo HS, Schneeweiss A, Barrios CH, Iwata $\mathrm{H}$, et al. Atezolizumab and nab-paclitaxel in advanced triple-negative breast cancer. $\mathrm{N}$ Engl $\mathrm{J}$ Med. 2018;379(22):2108-21.

2. Schmid P, Salgado R, Park YH, Munoz-Couselo E, Kim SB, Sohn J, et al. Pembrolizumab plus chemotherapy as neoadjuvant treatment of high-risk, early-stage triple-negative breast cancer: results from the phase $1 \mathrm{~b}$ open-label, multicohortKEYNOTE-173study. Ann Oncol. 2020;31(5):569-81.

3. Cortes J, Cescon DW, Rugo HS, Nowecki Z, Im SA, Yusof MM, et al. Pembrolizumab plus chemotherapy versus placebo plus chemotherapy for previously untreated locally recurrent inoperable or metastatic triple-negative breast cancer (KEYNOTE-355): a randomised, placebocontrolled, double-blind, phase 3 clinical trial. Lancet. 2020;396(10265):1817-28.

4. Robson M, Im SA, Senkus E, Xu B, Domchek SM, Masuda $\mathrm{N}$, et al. Olaparib for metastatic breast cancer in patients with a germline BRCA mutation. N Engl J Med. 2017;377(6):523-33.

5. Litton JK, Rugo HS, Ettl J, Hurvitz SA, Goncalves A, Lee KH, et al. Talazoparib in patients with advanced breast cancer and a germline BRCA mutation. N Engl J Med. 2018;379(8):753-63.

6. Stewart RL, Updike KL, Factor RE, Henry NL, Boucher KM, Bernard PS, et al. A multigene assay determines risk of recurrence in patients with triple-negative breast cancer. Cancer Res. 2019;79(13):3466-78.

7. Bardia A, Hurvitz SA, Tolaney SM, Loirat D, Punie K, Oliveira M, et al. Sacituzumab govitecan in metastatic triple-negative breast cancer. N Engl J Med. 2021;384(16): 1529-41.

8. Bardia A, Mayer IA, Vahdat LT, Tolaney SM, Isakoff SJ, DiamondJR, etal. Sacituzumabgovitecan-hziyin refractory metastatic triple-negative breast cancer. N Engl J Med. 2019;380(8):741-51.

9. Lipinski M, Parks DR, Rouse RV, Herzenberg LA. Human trophoblast cell-surface antigens defined by monoclonal antibodies. Proc Natl Acad Sci U SA. 1981;78(8):5147-50.

10. Lin H, Huang JF, Qiu JR, Zhang HL, Tang XJ, Li H, et al. Significantly upregulated TACSTD2 and cyclin D1 correlate with poor prognosis of invasive ductal breast cancer. Exp Mol Pathol. 2013;94(1):73-8.

11. Stein R, Chen S, Sharkey RM, Goldenberg DM. Murine monoclonal antibodies raised against human non-small cell carcinoma of the lung: specificity and tumor targeting. Cancer Res. 1990;50(4):1330-6.

12. SteinR, SharkeyRM, GoldenbergDM.Monoclonalantibody targeting of human non-small cell carcinoma of the lung. Cancer Res. 1990;50(3):866s-8s.

13. Goldenberg DM, Stein R, Sharkey RM. The emergence of trophoblast cell-surface antigen 2 (TROP-2) as a novel cancer target. Oncotarget. 2018;9(48):28989-9006.

14. Garrido-Castro AC, Keenan TE, Li T, Lange P, Callahan C, Guerriero J, et al. Saci-IO TNBC: randomized phase II trial of sacituzumab govitecan (SG) $+/$ - pembrolizumab in PD-L1-metastatictriple-negativebreastcancer (mTNBC). J Clin Oncol. 2021; https://doi.org/10.1200/JCO.2021.39. 15_suppl.TPS1106.

15. YardleyD, Abu-Khalaf M, BoniV, BrufskyA, Emens L, Gutierrez M, et al. Abstract OT2-06-04: MORPHEUS: a phase $\mathrm{Ib} / \mathrm{II}$ trial platform evaluating the safety and efficacy of multiple cancer immunotherapy combinations in patients with hormone receptor-positive and triple-negative breast cancer. Cancer Res. 2019;79(4):OT2-06-4-OT2-4.

16. Fabian KP, PadgetMR, Donahue RN, SolocinskiK, RobbinsY, Allen CT, et al. PD-Ll targeting high-affinity NK (t-haNK) cells induce direct antitumor effects and target suppressive MDSC populations. J Immunother Cancer. 2020;8(1):e450.

17. Yap TA, Hamilton EP, BauerTM, DumbravaEEI, Jeselsohn R, Enke A, et al. 547P rucaparib + sacituzumab govitecan (SG): Initial data from the phase Ib/II SEASTAR study (NCT03992131). Ann Oncol. 2020;31:S476-S7.

18. Marmé F, Stickeler E, Furlanetto J, Denkert C, Schmidt M, Reinisch M, et al. Phase III postneoadjuvant study evaluating sacituzumab govitecan, an antibody drug conjugate in primary HER2-negative breast cancer patients with high relapse risk after standard neoadjuvant treatment: SASCIA. JClin Oncol. 2021;39(15):TPS602-TPS.

19. Spring LM, Tolaney SM, Desai N, Comander A, Mulvey T, Krop IE, et al. Phase 2 study of response-guided neoadjuvant sacituzumab govitecan (IMMU-132) in patients with localized triple-negative breast cancer (NeoSTAR). Cancer Res. 2021; https://doi.org/10.1158/1538-7445.SABCS20OT-03-06. 


\section{short review}

20. Modi S, Saura C, Yamashita T, Park YH, Kim SB, Tamura K, et al. Trastuzumab deruxtecan in previously treated HER2positive breast cancer. NEngl J Med. 2020;382(7):610-21.

21. Okajima D, Yasuda S, Maejima T, Karibe T, Sakurai K, Aida T, et al. Datopotamab deruxtecan, a novel TROP2-directed antibody-drug conjugate, demonstrates potent antitumor activity by efficient drug delivery to tumor cells. Mol Cancer Ther. 2021; https://doi.org/10.1158/1535-7163.MCT-210206.

22. Yoh K, Goto Y, Thomas M, Spira AI, Sands J, Johnson ML, et al. A randomized, phase 3 study of datopotamab deruxtecan (Dato-DXd; DS-1062) versus docetaxel in previously treated advanced or metastatic non-small cell lung cancer (NSCLC) without actionable genomic alterations (TROPION-Lung01). J Clin Oncol. 2021;39(15):TPS9127TPS.

23. Bardia A, Juric D, Shimizu T, Tolcher A, Karim R, Spira A, et al. Datopotamab deruxtecan (Dato-DXd), a TROP2-directed antibody-drug conjugate (ADC), for triple-negative breast cancer (TNBC): preliminary results from an ongoing phase I trial. Ann Oncol. 2021;32:S60.

24. Liu Y, Lian W, Zhao X, Diao Y, Xu J, Xiao L, et al. SKB264 ADC: a first-in-human study of SKB264 in patients with locally advanced unresectable/metastatic solid tumors who are refractory to available standard therapies. J Clin Oncol. 2020;38(15):TPS3659-TPS.

25. King GT, Eaton KD, Beagle BR, ZopfCJ, Wong GY, Krupka HI, et al. A phase 1, dose-escalation study of PF-06664178, an anti-Trop-2/Aur0101 antibody-drug conjugate in patients with advanced or metastatic solid tumors. Invest New Drugs. 2018;36(5):836-47.

Publisher's Note Springer Nature remains neutral with regard to jurisdictional claims in published maps and institutional affiliations.

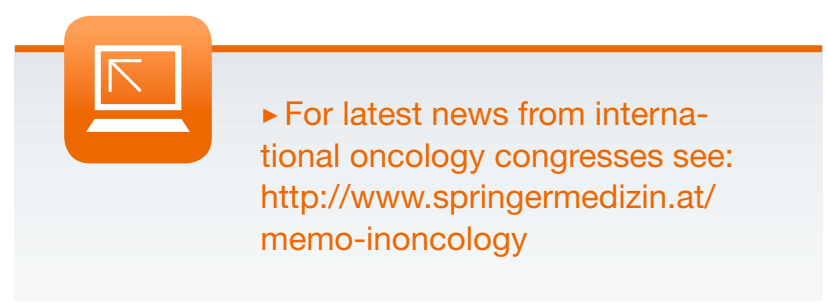

